\title{
ON THE CIRCULAR POLARIZATION IN ACTIVE REGIONS
}

\author{
E. WIEHR
}

Universitäts-Sternwarte, Göttingen, Germany

\begin{abstract}
Advantages of 'circular polarimetry' (determination of the Stokes parameter V) in active regions as compared to 'elliptical polarimetry' (determination of all Stokes parameters) are discussed:
\end{abstract}

(1) Complicated and doubtful reduction procedures by means of line formation theory generally are needed for 'elliptical polarimetry'. This is not the case for 'circular polarimetry'.

(2) Even if the difficulties (1) could be removed, 'elliptical polarimetry' would not help to determine the total magnetic vector outside the disc center. This is due to the fact that the azimuth $\varphi$ of the linear polarization is only defined for $0^{\circ} \leqslant \varphi<180^{\circ}$. When converting the measured inclination $\psi$ to the line-of-sight into the true inclination $\gamma$ to the solar surface normal for active regions outside the disc center, the ambiguity of $\varphi$ always yields an ambiguity of $\gamma$. The normal field component and thus the flux can therefore not be determined without making an important assumption on the unknown field structure (see Wiehr, 1970a).

(3) For active regions near the disc center, however, where the difficulty (2) disappears, 'circular polarimetry' is sufficient for the determination of the normal field component and flux. It furthermore avoids the difficulty (1).

Some useful applications of such 'circular polarimetry' near the disc center are mentioned:

(a) Exact determinations of the magnetic flux for several active regions near the disc center show that the disbalances between the flux in preceding and following regions are within the error limits when using a Zeeman line which remains unchanged going from photosphere to spot (e.g. Fe $\lambda$ 6302.5). The often reported flux disbalances (Stenflo, 1967) as observed in $\mathrm{Fe} \lambda 5250$ or $\mathrm{H} \alpha$, therefore, seem to be mainly due to the differences between the line absorption coefficients in the photosphere (where the calibration is carried out) and the spots or faculae (where the magnetic fields are measured).

(b) Differences between the normal field components as obtained from 'circular polarimetry' in different lines (Severny and Bumba, 1958) seem to be doubtful if the line profile changes mentioned in (a) are not taken into account.

(c) The development of active regions near the disc center can be studied very well by means of 'circular polarimetry'. An example for a young active region is shown. The development of the normal field component generally confirms the observations by Gopasyuk (1967). The development of the flux had to be extrapolated backward in time since the very beginning of this region unfortunately was not observed. (For details see Wiehr, 1970b.)

Further observations of this kind could yield important information on the first 
occurrence and the development of active regions as well as possible correlations with flares.

\section{References}

Gopasyuk, S. I.: 1967, Publ. Crim. Astron. Obs. 36, 56.

Severny, A. B. and Bumba, V.: 1958, Observatory 78, 33.

Stenflo, J. O.: 1968, in K. O. Kiepenheuer (ed.), 'Structure and Development of Solar Active Regions', IAU Symp. 35, 47.

Wiehr, E.: 1970a, Solar Phys. 11, 399.

Wiehr, E.: 1970b, Solar Phys. 15, 148.

\section{Discussion}

Giovanelli: Is there any intrinsic difficulty in calibrating a longitudinal magnetograph by measuring the slope of the line profile at the point on the solar disk where the field is being measured?

Wiehr: In principal such a calibration is possible, but in practice it would require us to measure point by point the polarization and line profile on the solar disk. This would extend the already long observation time by at least a factor two. 